\title{
Medición de los niveles de ruido ambiental en la ciudad de Santiago de Chile
}

\section{Environmental noise levels measurement of the city of Santiago, Chile}

\author{
Lisbeth Platzer $\mathrm{M}^{1}$, Rodrigo Iñiguez $\mathrm{C}^{2}$, Jimena Cevo $\mathrm{E}^{3}$, Fernanda Ayala $\mathrm{R}^{3}$.
}

\begin{abstract}
RESUMEN
Introducción. Un estudio realizado en Santiago en 1989, estimó que 1.300.000 personas estaban sometidas a niveles de ruido inaceptables por las normas internacionales. Considerando que no existen publicaciones sobre ruido ambiental realizadas por otorrinolaringólogos, y que el tema no ha sido revisado en los últimos 15 años, quisimos actualizar la información al respecto.

Material y método. Se evaluó el ruido en lugares que afectan la rutina del ciudadano común, independiente de su profesión: Barrio residencial, parques, discotecas, bardiscoteque, calles principales, buses de transporte urbano habituales, buses de transporte urbano del proyecto transantiago y Metro. Las mediciones se realizaron con un sonómetro integrador, según lo establecido por la normas de la Comisión Nacional del Medio Ambiente (CONAMA), durante los meses de julio a septiembre del 2006.

Resultados. Las discotecas son el lugar con mayor contaminación acústica. En relación a la Avda. Bernardo O'Higgins (Alameda), el nivel de ruido: $80.5 \mathrm{~dB}(\mathrm{~A})$, se mantiene constante en diferentes puntos de medición. Entre el transporte público, el metro tiene los niveles de ruido más altos: $87 d B(A)$. No existen diferencias entre los buses antiguos o "micros amarillas" y los del proyecto transantiago.

Conclusiones. Nuestros resultados ponen una nota de alerta mostrando que el nivel de ruido en Santiago, para la gran mayoría de los parámetros, son superiores a las normas establecidas.
\end{abstract}

Palabras claves: Ruido ambiental, contaminación acústica, trauma acústico, transporte urbano.

\begin{abstract}
Introduction. A study carried out in Santiago in 1989 estimated that 1,300,000 people were exposed to noise levels unacceptable by international standards. Considering that there are no reports of environmental noise evaluations performed by
\end{abstract}

Residente Otorrinolaringología, Pontificia Universidad Católica de Chile.

Instructor asociado, Departamento de Otorrinolaringología, Pontificia Universidad Católica de Chile.

Médico cirujano. Pontificia Universidad Católica de Chile. 
otorhinolaryngologists, and that the subject has not been reviewed in the past 15 years, in this paper we present up-to-date information on the topic.

Materials and methods. Noise levels were evaluated in several places that are part of the common citizen life, disregarding their profession: Residential neighborhoods, parks, discos, pubs, main streets, city buses (old and new system), and subway. Measurements were taken using an integrating sound level meter, according to CONAMA standards, between the months of July and September, 2006.

Results. The highest noise pollution was found in discos. With regard to the main avenue in Santiago, Libertador B. O'Higgins, noise levels (80 dB (A)) remained constant in different measured points. As of public transportation, the subway had the higher noise levels (87 $d B(A))$. There were no differences between the old and new public transit buses.

Conclusions. Our results raise an environmental alert, showing that the noise level in Santiago, for most parameters, is above international standards.

Key words. Environmental noise, noise pollution, acoustic trauma, urban public transportation.

\section{INTRODUCCIÓN}

El ruido ambiental se ha convertido en uno de los contaminantes más molestos de la sociedad moderna que incide directamente sobre el bienestar de la población. Las personas sometidas a grandes ruidos de forma continua, experimentan serios trastornos fisiológicos, como pérdida de la capacidad auditiva, alteración de la actividad cerebral, cardiaca y respiratoria, trastornos gastrointestinales, entre otros. Además se producen alteraciones conductuales tales como perturbación del sueño y el descanso, dificultades para la comunicación, irritabilidad, agresividad, problemas para desarrollar la atención y concentración mental ${ }^{1,2}$. En la unión europea la contaminación acústica afecta unos 100 millones de personas y causa pérdidas por más de 60 millones de euros 3 .

De las fuentes de ruido urbano, los vehículos motorizados son responsables de aproximadamente el $70 \%$ del ruido presente en las ciudades, y de él, el mayor aporte lo representan los vehículos de mayor tamaño, entre ellos la locomoción colectiva. Un segundo grupo lo constituyen las «fuentes fijas», es decir, las industrias, construcción, talleres, centros de recreación, etc. Los agentes de menor impacto son aquellos de ocurrencia esporádica como: gritos de los niños, conciertos al aire libre, ferias y vendedores callejeros, sonidos de animales domésticos, fuegos artificiales, etc ${ }^{4,5}$.
La unidad de medida del sonido es el decibel $(\mathrm{dB})$ y el instrumento que se utiliza para medir el ruido es el sonómetro. El indicador más fácil para medir el ruido ambiental es el nivel de presión sonora (NPS) expresado en $\mathrm{dB}$ y corregido por el filtro de ponderación (A), que permite que el sonómetro perciba las frecuencia $(\mathrm{Hz})$ de manera similar a como los escucha el oído humano (NPS $d b(A))^{6}$. Se sabe que el daño acústico es proporcional tanto a la intensidad del sonido como al tiempo de exposición. Es por ello que la norma chilena establece como un periodo máximo de exposición laboral de 8 horas a $85 \mathrm{~dB}(\mathrm{~A}), 4$ horas a $90 \mathrm{~dB}(\mathrm{~A}) 02$ horas a $95 \mathrm{~dB}(\mathrm{~A})^{7}$.

La Organización Mundial de la Salud (OMS) ha sugerido un valor de ruido de $55 \mathrm{~dB}(\mathrm{~A})$ como límite superior deseable al aire libre. Se sugieren valores

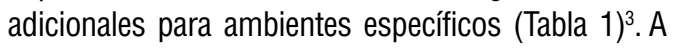

Tabla 1. Niveles de ruido sugeridos por la OMS para ambientes específicos

\begin{tabular}{|lc|}
\hline Ambientes & $\mathrm{dB}(\mathrm{A})$ \\
\hline Viviendas & $50 \mathrm{~dB}(\mathrm{~A})$ \\
Escuelas & $35 \mathrm{~dB}(\mathrm{~A})$ \\
Discotecas & $90 \mathrm{~dB}(\mathrm{~A}) \times 4 \mathrm{~h}$ \\
Conciertos, Festivales & $100 \mathrm{~dB}(\mathrm{~A}) \times 4 \mathrm{~h}$ \\
Comercio y tráfico & $70 \mathrm{~dB}(\mathrm{~A})$ \\
\hline
\end{tabular}


través de las Normas ISO² (International Organization for Standardization), el cual es un organismo que emite normas internacionales luego del trabajo de varios comités técnicos y votación de sus miembros, se ha sugerido que niveles de ruido inferiores a 70 $\mathrm{dB}(\mathrm{A})$ durante las 24 horas del día, no produciría deficiencias auditivas. Para los ruidos imprevistos se propone que el nivel de presión sonora (NPS) nunca debe exceder los $140 \mathrm{~dB}$ para adultos y $120 \mathrm{~dB}$ para niños ${ }^{8}$.

Un estudio realizado en el año 1989 por la Universidad de Santiago (USACH), sobre las bases de generación de ruido en la ciudad de Santiago de Chile, estimó que aproximadamente 1.300 .000 personas estaban sometidas a niveles de ruido considerados inaceptables por las normas de calidad ambiental internacionales ${ }^{9}$. Buscando reducir el problema, el Ministerio de Transportes y la Comisión Nacional del Medio Ambiente (CONAMA), han establecido normas de emisión de ruido para buses de locomoción colectiva urbana y rural y también para la emisión de ruidos molestos generados por fuentes fijas.

Los valores límites para la emisión de ruidos molestos generados por fuentes fijas, han sido estimados según se informa en la Tabla 2 y se han agrupado en zonas de acuerdo a los instrumentos de planificación territorial:

- La zona I: corresponde a habitacional y equipamiento a escala vecinal.

- La zona II corresponde a equipamiento a escala comunal y/o regional.

- La zona III: permite industria inofensiva.

- La zona IV: permite a industria inofensiva y/o molesta $^{10}$.

El aumento en la actividad y crecimiento del país, hace suponer que la contaminación acústica

Tabla 2: Niveles máximos permisibles de presión sonora corregidos (NPC) en $\mathrm{db}(a)$ lento, según CONAMA

\begin{tabular}{|lcc|}
\hline & de 7 a 21 Hrs. & de 21 a 7 Hrs. \\
\hline Zona I & 55 & 45 \\
Zona II & 60 & 50 \\
Zona III & 65 & 55 \\
Zona IV & 70 & 70 \\
\hline
\end{tabular}

es un problema cada vez más frecuente. A pesar de lo anterior, en los últimos 15 años hay muy pocos trabajos que investiguen acerca del tema, la realidad nacional actual y su efecto en las personas. En el presente trabajo de investigación, se pretende reflejar de la forma más fidedigna posible, la realidad de un problema que afecta a la sociedad en general, en especial a aquellas personas que viven en grandes núcleos urbanos. Además, busca actualizar la información al respecto, considerando que no existen publicaciones sobre ruido ambiental realizadas por otorrinolaringólogos.

\section{OBJETIVOS}

1. Describir los niveles de ruido ambiental en la ciudad de Santiago, en zonas que afectan la rutina del ciudadano común, independiente de su profesión.

2. Comparar el resultado de las mediciones con las normas nacionales, internacionales y estudios previos.

3. Difundir los resultados del estudio en nuestro medio, con el fin de hacer conciencia sobre la contaminación acústica y el eventual daño al que estamos expuestos diariamente.

\section{METODOLOGÍA}

Se realizó un estudio descriptivo, transversal, durante junio-septiembre 2006. Las mediciones se realizaron entre las 16:00 y las 20:00 horas, exceptuando bar-discoteques que fueron evaluados entre las 21:00 y 2:00 horas.

\section{Instrumento de medición}

Se utilizó un sonómetro integrador tipo 2 (margen error $\pm 1 \mathrm{~dB}$ ), debidamente calibrado, que cumplía con las exigencias señaladas para los tipos 0,1 ó 2 , establecidas en las normas de la Comisión Electrotécnica Internacional (International Electrotechnical Commission, IEC Standard), publicaciones № 651 Sonómetros (Sound Level Meters), primera edición de 1979; y № 804 Sonómetros Integradores-promediadores (Integrating-averaging Sound Level Meters), primera edición de $1985^{10}$. 
Se utilizó el filtro de ponderación A y la respuesta lenta del instrumento de medición. Los resultados de las mediciones se expresaron en $\mathrm{dB}(\mathrm{A})$ lento.

\section{Condiciones de medición}

Se efectuaron según lo establecido por la normas de medición de ruido de CONAMA ${ }^{11}$. Los puntos de medición se ubicaron entre 1,2 y 1,5 metros sobre el suelo, y en caso de ser posible, a unos 3,5 metros 0 más de las paredes, construcciones $u$ otras estructuras reflectantes. Se efectuarán como mínimo tres mediciones de 1 minuto duración, en puntos separados entre sí en 0,5 metros aproximadamente, y de ellas se obtuvo el promedio aritmético. Se descartó las mediciones que incluyeron ruidos ocasionales.

\section{Fuentes de ruido medidas}

Se evaluó el ruido ambiental emitido en zonas que afectan la rutina del ciudadano común independiente de su profesión, por ello los lugares de medición han sido elegidos en forma arbitraria:

- Dentro de las zonas de emisión de ruido de fuentes fijas se registró: Barrio residencial (zona 1), centros recreacionales, parques, discotecas, bar-discoteques (zona 2-3).

- Además decidimos evaluar la emisión de ruido existente en las calles con mayor emisión de ruido en Santiago.

- En cuanto al registro del ruido emitido por el transporte público se realizó en bus convencional (micro amarilla), bus del proyecto transan- tiago y metro. Para ello se realizaron mediciones dentro de los buses y el metro, así como también en andenes. Se realizó un promedio de mediciones al sentarse atrás, al medio y adelante de cada transporte.

\section{RESULTADOS}

Al evaluar las fuentes de emisión fija de ruido, se encontró que el barrio residencial de Pedro de Valdivia Norte (que fue escogido por la ausencia de locomoción colectiva), presentaba niveles de ruido promedio de $57,5 \mathrm{~dB}(\mathrm{~A})$, lo que es superior al máximo permitido para una zona 10 residencial (Figura 1). Asimismo, al evaluar las zonas 2-3, las mediciones sólo arrojaron valores sobre el máximo permitido, y encontramos que tanto bares como discotecas incluso llegan a doblar dicho valor. Al realizar un registro de un parque, este también arrojaba valores cercanos al límite sugerido por la norma (Figura 2).

En relación a la principal calle de Santiago, Avda. Bernardo 0'Higgins (Alameda), el nivel de ruido se mantiene en general constante en los diferentes puntos de medición, siendo el promedio de 82 $d B(A)$, con un rango de 79-87 dB(A) (Figura 3). Dentro de las mediciones efectuadas al transporte público de Santiago, el Metro fue el que tuvo los niveles de ruido más altos: para un viaje dentro del vagón de linea 1 se obtuvo un valor promedio de 87,1 $\mathrm{db}(\mathrm{A})$ y para mediciones en el andén de las estaciones, el nivel de ruido fluctuaba entre $80-85 \mathrm{~dB}(\mathrm{~A})$. Las mediciones registradas en las micros amarillas

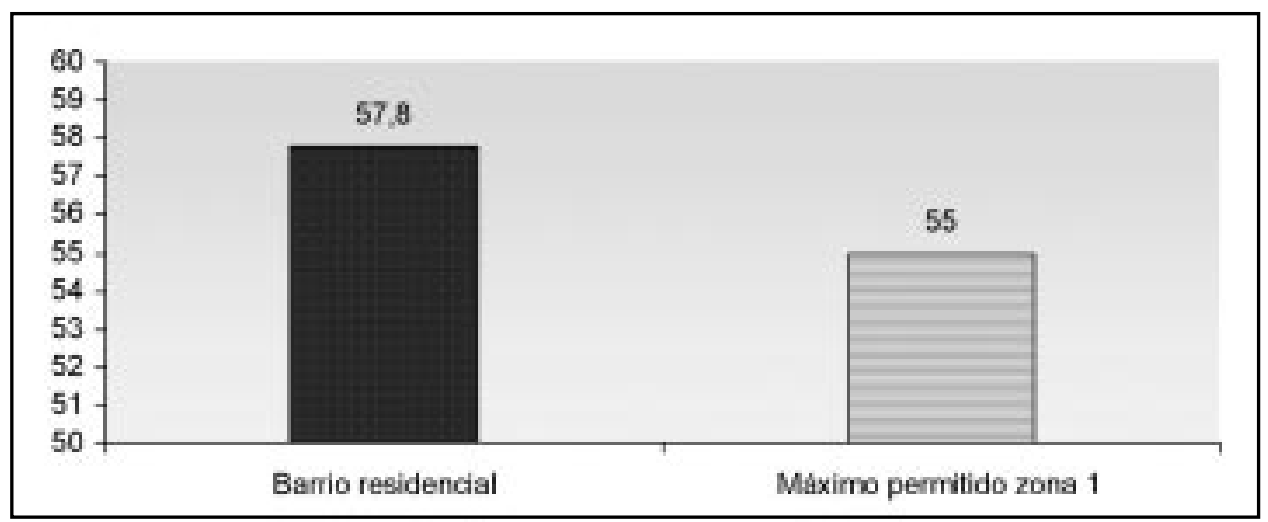

Figura 1: Niveles de ruido medidos en zona 1 (escala vecinal). 


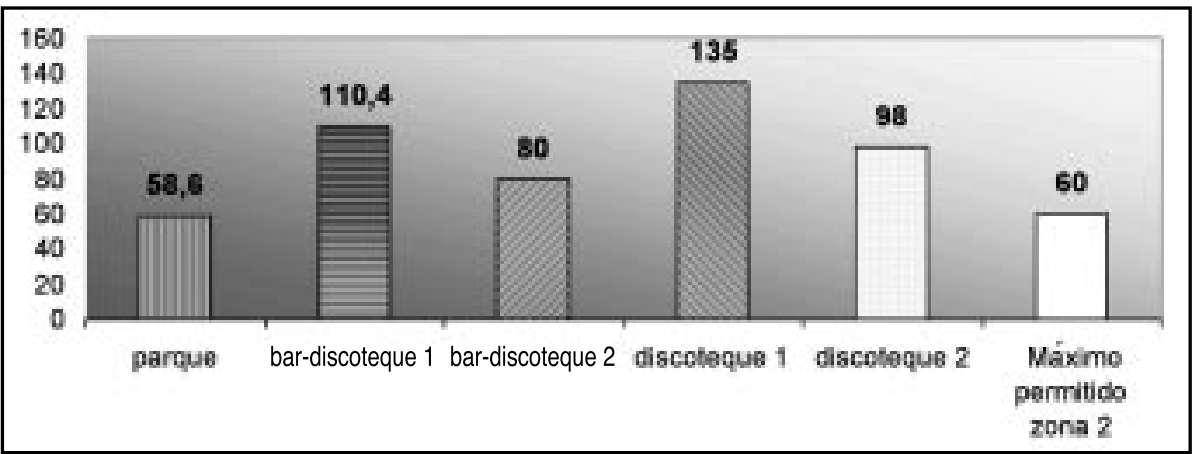

Figura 2: Niveles de ruido medidos en zona 2-3.

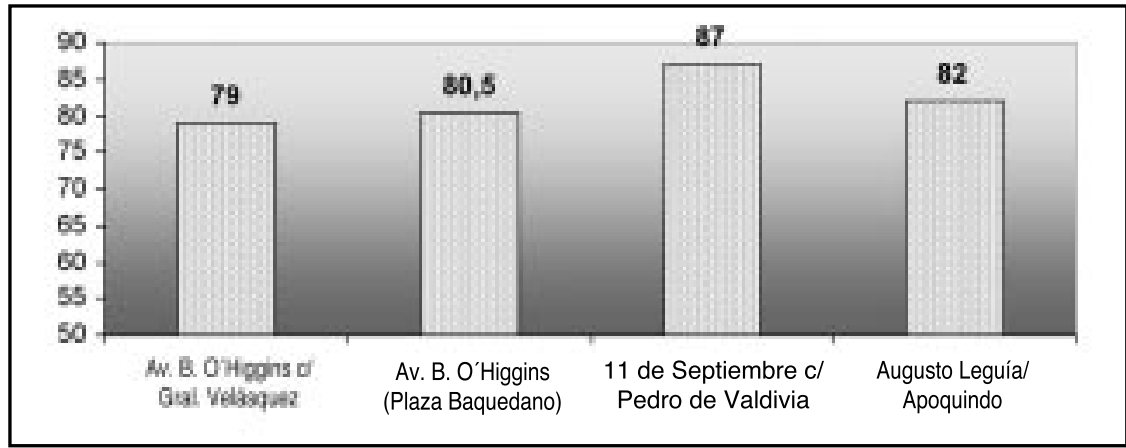

Figura 3: Niveles de ruido medidos en esquinas de la principal calle de Santiago: Avda. Bernardo 0’Higgins (Alameda) y su prolongación al oriente (11 de Septiembre y Apoquindo).

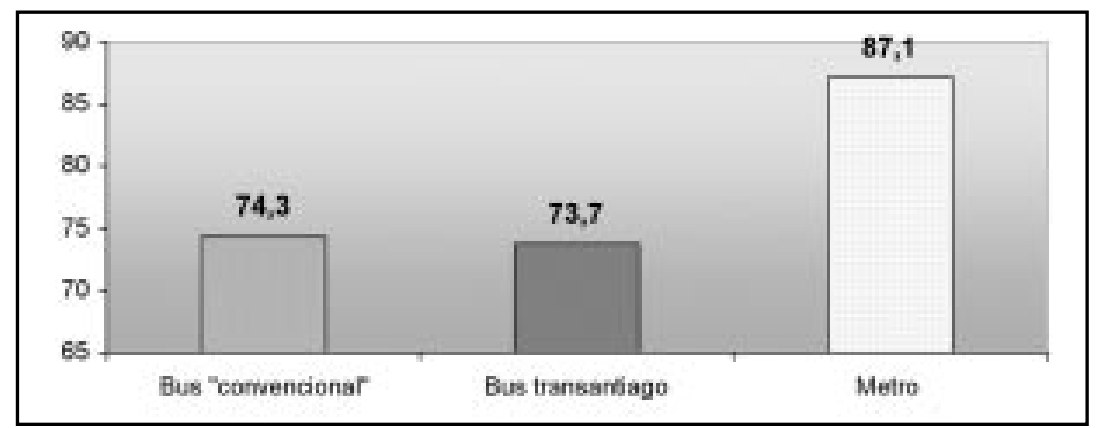

Figura 4: Niveles de ruido medidos en el transporte público.

convencionales fue de $74 \mathrm{db}(\mathrm{A})$ y en los buses del proyecto transantiago $73 \mathrm{db}(\mathrm{A})$ (Figura 4).

\section{DISCUSIÓN}

La contaminación acústica es considerada por la mayoría de la población de las grandes ciudades como un factor medioambiental muy importante, que incide de forma principal en su calidad de vida. Como ejemplo, podemos señalar que a nivel de Servicio Metropolitano Ambiental (SESMA) en la Región Metropolitana, se estima en cerca de 720 denuncias por ruidos molestos ocasionados por fuentes fijas al año. A nivel municipal, se estima que cerca del $50 \%$ del total de denuncias corres- 
ponde a demandas tipificables como de fuentes fijas $^{11}$.

En comparación con otros contaminantes, el control del ruido ambiental se ha limitado por la falta de conocimiento de sus efectos sobre los seres humanos, la escasa información sobre la relación dosis-respuesta y la falta de criterios definidos. Nuestros resultados muestran que las discotecas son el lugar con mayor contaminación acústica, lo cual es preocupante dado el tipo de población que asiste. Los valores obtenidos sobrepasan todas las normas internacionales. Si bien los barrios residenciales tienen menor cantidad de ruido que los espacios con mayor afluencia de público, igualmente sobrepasan la norma establecida para la Zona 10 residencial.

Estudios realizados en 1979 por la USACH ${ }^{4}$ y en 1989 por el SESMA, describían que el ruido de tránsito fluctuaba entre 65-71 dB A, siendo la calle con mayor medición de ruido de 78,6 db $(A)^{10}$. En general, las mediciones obtenidas en nuestro estudio son más altas que la de años anteriores ${ }^{4,5,13,14}$ Io que puede explicarse probablemente por un aumento en el número de vehículos. Al evaluar los niveles de ruido a lo largo de la Avda. Bernardo 0’Higgins (Alameda), principal calle de nuestra ciudad, encontramos que no existe una gran diferencia en la emisión de ruido ambiental a través de sus distintos tramos, con un discreto aumento en las estaciones de registro más céntricas. Si comparáramos cualquiera de estas esquinas registradas, con el máximo nivel de ruido permitido para una zona industrial 0 zona $4(70 \mathrm{db}(\mathrm{A}))$, todas ellas estarían por sobre este máximo.

En relación a los niveles de ruido que está expuesto un pasajero que hace uso del transporte público, todas las mediciones superan el máximo permitido de $60 \mathrm{~dB}(\mathrm{~A})$ para zonas industriales. Sin embargo, llama la atención que los valores obtenidos, no sobrepasan los niveles permitidos por la CONAMA para el interior de la locomoción colectiva, los que están considerados en $79 \mathrm{db}$ en condiciones dinámicas ${ }^{8}$, como tampoco sobrepasan los niveles de presión sonora máximo permitidos para la exposición ocupacional de trabajadores expuestos sin protección auditiva, considerados en $80 \mathrm{~dB}$ para un tiempo de exposición de $24 \mathrm{hrs}$. En relación a este punto es discutible que los valores permitidos para el interior de los buses sean
Tabla 3: Comparación de mediciones de ruido efectuadas al metro en año 1981 y 2006

\begin{tabular}{|lrr|}
\hline Metro & 1981 & 2006 \\
\hline Línea 1 (anden) & $86,2 \mathrm{~dB}(\mathrm{~A})$ & $87,1 \mathrm{~dB}(\mathrm{~A})$ \\
Ruido de fondo & $70 \mathrm{~dB}(\mathrm{~A})$ & $68 \mathrm{~dB}(\mathrm{~A})$ \\
\hline
\end{tabular}

considerablemente más altos que los exigidos para la industria, considerando la cantidad de población que es directamente afectada.

Dentro del transporte público, el Metro es quien tiene los niveles de ruido más altos (Figura 4). Si comparáramos esta medición, con un estudio realizado en 1981 por la USACH (Tabla 3), vemos que los niveles se han mantenido en general estables en los últimos 25 años. Ya en ese entonces, se concluía que los niveles encontrados sobrepasaban todos los límites recomendados, pese a lo cual no ha habido cambios. Creemos que estas cifras deberían ser consideradas, no sólo por la afectación que sufrirían los pasajeros, sino también, por que existen conductores que están expuestos diariamente a estos niveles de ruido. No se evidencia gran diferencia en el nivel de ruido emitido entre buses del proyecto transantiago y las "micros amarillas" o buses convencionales.

\section{CONCLUSIÓN}

Nuestros resultados ponen una nota de alerta mostrando que el nivel de ruido en la ciudad de Santiago, para la gran mayoría de los parámetros, es superior a las normas establecidas. Falta aún elaborar estudios que muestren la relación entre la contaminación acústica y posibles alteraciones de salud en la población nacional general, tanto físicas como psicológicas.

\section{BIBLIOGRAFÍA}

1. Donath T. Effects of environmental noises and sounds on the quality of life controlled by the central nervous system. Orvosi Hetilap. 2006 Apr 3; 147 (16):737-9. 
2. ISO 1999:1990 (E). "Acoustics-Determination of occupational noise exposure and estimation of noise-induced hearing impairment». International Organization for Standardization, Genève, Suiza, 1990.

3. Guías para el ruido urbano-Documento guía al Department of the Protection of the Human Environment, Occupational and Environmental Health, Organización Mundial de la Salud, Ginebra, Suiza 1995 http://www.who.int/ environmental_information.

4. Hernán Costabal T, Faúndez Abans. Estudio contaminación acústica en Santiago Chile. USACH 1979 area fisica 9 (34): 15-29.

5. Darío Mosca. Contaminación Acústica Originada por el Ruido del Tránsito y Proposiciones para su Evaluación en el Área Metropolitana. Informe Técnico. MINSAL. Santiago, 1980.

6. Asociación Chilena de Municipalidades, Fundación Alemana para el Desarrollo Internacional; Fundación Friedrich. Gestión ambiental Municipal. Santiago de Chile, 1995, octubre pag 158-71.

7. Reglamento sobre condiciones sanitarias y ambientales básicas en los lugares de trabajo. CONAMA Decreto supremo N 594/1999.
8. Norma de Emisión de Ruido para Buses de Locomoción Colectiva Urbana y Rural. CONAMA Decreto supremo N 129/02 del Ministerio de Transporte y Telecomunicaciones.

9. Estudio Base de Generación de Niveles de Ruido. Proyecto de Descontaminación Ambiental Región Metropolitana. Contratante: Intendencia R.M. Consultor: Depto. de Física, USACH, 1989.

10. Corporación Nacional de Medio Ambiente, Chile 2006. Www. CONAMA. cl

11. Manual de aplicación norma de emisión de ruidos molestos generados por fuentes fijas. CONAMA D.S no 146/1997 del Ministerio Secretaría General de la Presidencia.

12. Normas de la Comisión Electrotécnica Internacional (IEC), publicaciones № 651 y № $804.1^{1}$ Ed 1985.

13. Jorge Lay Gajardo, Iván Milos, R Pesse Lohr et al. Medio ambiente acústico en metro de Santiago. Contribuciones científicas y tecnológicas Físicas 1981, 11 (50): 29-38.

14. Varas M, Hernán. Epidemiología del ruido comunitario en comuna de Providencia. U. De Chile 1994. 